\title{
Multichannel Quantum-Defect Study of $q$ reversals in Overlapping Resonances in Systems involving 1 Open and 2 Closed Channels
}

\author{
Byunghoon Cho and Chun-Woo Lee* \\ Department of Chemistry, Ajou University, Suwon 443-749, Korea. *E-mail: clee@ajou.ac.kr \\ Received November 17, 2009, Accepted December 11, 2009
}

\begin{abstract}
This study examined the overlapping resonances in the systems involving 1 open and 2 closed channels using the phaseshifted version of multichannel quantum-defect theory (MQDT). The results showed that 21 patterns for the $q$ reversals in the autoionization spectra are possible depending on the relative arrangements of the two simple poles and roots of the quadratic equations. Complete cases could be generated easily using the $q$ zero planes determined using only 3 asymmetric spectral line profile indices. The transition of the spectra of the coarse interloper Rydberg series from the lines into a structured continuum by being dispersed onto the entire Rydberg series was found. The overall behavior of the time delays was found to be governed by the dense Rydberg series, which is quite different from the one of the autoionization cross sections that is governed by an interloper, indicating that different dynamics prevail for them. This is in contrast to the two channel system where both quantities behave similarly. The dynamics obtained in the presence of overlapping resonances is as follows. The absorption process is instant and dominated by a transition to the interloper line. This process is followed by rapid leakage into the dense Rydberg series, which has a longer residence time before ionization than that of the interloper state. This is because the orbiting period is proportional to $v^{3}$ so that an excited electron has a shorter lifetime in the interloper state belonging to a lower member of the Rydberg series.
\end{abstract}

Key Words: Phase-shifted MQDT, Overlapping resonance, $q$ reversal

\section{Introduction}

Complex overlapping resonances caused by an interloper are observed routinely in autoionization spectra, such as in $\mathrm{N}_{2}$, $\mathrm{Ba}^{2} \mathrm{~Pb}^{3}$ and $\mathrm{Yb}^{4}$ Although configuration mixing theory $(\mathrm{CM})$ was the first to study overlapping resonances in atomic and molecular systems, ${ }^{5-6}$ there have been several difficulties in implementing it and only a few studies in this line of research have been carried out. ${ }^{7}$ Multichannel quantum defect theories (MQDT), ${ }^{8-9}$ with the implementation of phase renormalization, ${ }^{10-11}$ are extremely powerful for dealing with observed overlapping resonances. ${ }^{12}$ Dealing only 3 channel systems with one open and 2 closed, or occasionally one more open channel being included, ${ }^{13-14}$ was sufficient to reproduce the numerous overlapping resonances observed. Fundamental studies of these systems particularly for the perturbed Rydberg series with a single closed channel acting as an interloper were performed by GiustiSuzor and Lefebvre-Brion, ${ }^{15}$ Cooke and Cromer, ${ }^{11}$ Wintgen and Friedrich ${ }^{16}$ and Ueda. ${ }^{17}$ They examined the phenomena in complex resonances such as intensity borrowing, $q$ reversals and truly bound states in continuum. In addition to their studies, work by Lane $e t a l .^{3}$ should be mentioned despite the defect in the method that scattering theories were used to examine the autoionization spectra. Nonetheless, the systems showed a range of interesting aspects of overlapping resonances and are worth adopting in the present work. In another study, ${ }^{7}$ they adapted Fano's CM theory to handle the interloper in overlapping resonances. This work was applied once by Gallagher's group to explain their experimental data. ${ }^{18}$

Giusti-Suzor and Lefebvre-Brion ${ }^{15}$ were the first to lay the foundation for studies of the complex resonance phenomena caused by an interloper. Their formulation of the influence of an interloper in terms of direct and indirect processes is full of physical insights and worth further study. Cooke and Cromer ${ }^{11}$ derived the formulae for the widths and shifts of the peaks in the Rydberg series due to an interloper. Their formulation contained almost all the ingredients of Ueda's one ${ }^{17}$ but was limited to the isolated core excitation (ICE) spectra. Their formula lacked an integration of various parts into a single form. In contrast to Cooke and Cromer, Ueda's formulation dealt directly with the cross section itself and decomposed it into the product of Beutler-Fano functions corresponding to the interloper term appearing as an envelope one and the dense Rydberg series appearing as a chopper of the Rydberg series of the interloper. Wintgen and Friedrich ${ }^{16}$ also dealt directly with the cross section. They instead formulated it into one Beutler-Fano form. Ueda's formulation has the advantage of disentangling the physical terms of a different nature, each of which behaves much simpler and provides easy access to the dynamics of a complex resonance. In its nascent form, it has a limitation. Therefore, this study examined the pole structure of the principal part of Ueda's formula and utilized it to disclose all possible dynamics that bring about a $q$ reversal with the help of the root surfaces of $q$.

Summary of formulas due to Ueda and others. Currently, the practical use of multichannel quantum defect theory (MQDT) almost always adopts the phase-shifted MQDT and starts directly from either zero submatrices or zero diagonal submatrices depending on the types of system. ${ }^{12}$ According to this practice, the short-range reactance matrix takes the following form for systems involving 1 open and 2 closed channels:

$$
\tilde{K}=\left(\begin{array}{cc}
\tilde{K}^{o o} & \tilde{K}^{o c} \\
\tilde{K}^{c o} & \tilde{K}^{c c}
\end{array}\right)=\left(\begin{array}{ccc}
0 & \tilde{K}_{12} & \tilde{K}_{13} \\
\tilde{K}_{12} & 0 & \tilde{K}_{23} \\
\tilde{K}_{13} & \tilde{K}_{23} & 0
\end{array}\right)
$$


In the energy range where one channel is open and 2 other channels are closed, index 1 will be used for the open channel and 2 and 3 will be used for the closed ones. Let us assume that the ionization thresholds $I_{i}$ satisfy $I_{1}<I_{2}<I_{3}$. If the energy-normalized eigenfunction $\boldsymbol{\Psi}$ is expanded into

$$
\boldsymbol{\Psi}=\sum_{i} \tilde{\Psi}_{i} \tilde{Z}_{i} \cos \pi\left(v_{i}+\mu_{i}\right)
$$

in terms of the standing-wave channel basis functions, $\tilde{\Psi}_{i}$ can be decoupled outside the reaction zone as follows:

$$
\tilde{\Psi}_{i}=\sum_{j=1}^{3} \Phi_{j}(\omega)\left[\tilde{f}_{j}(R) \delta_{j i}-\tilde{g}_{j}(R) \tilde{K}_{j i}\right],\left(R>R_{0}\right),
$$

where $\left(\tilde{f}_{j}, \tilde{g}_{j}\right)$ denotes the phase-shifted regular and irregular base pair

$$
\begin{aligned}
& \tilde{f}_{j}=f_{j} \cos \pi \mu_{j}-g_{j} \sin \pi \mu_{j} \\
& \tilde{g}_{j}=g_{j} \cos \pi \mu_{j}+f_{j} \sin \pi \mu_{j}
\end{aligned}
$$

for motion along the ionization coordinate $R$, and $\Phi_{j}(\omega)$ are the wavefunctions composed of the ion core and angular and spin part of the outer electron in the $j^{\text {th }}$ channel. The regular and irregular base pair $\left(f_{j}, g_{j}\right)$ belongs to the $j^{\text {th }}$ ionization threshold energy $I_{j}$ with an effective quantum number $v_{j}$ given by $E=$ $I_{j}-$ Ryd $/ v_{j}^{2}$. The expansion coefficients in (2) can be obtained as follows:

$$
\begin{aligned}
& \tilde{Z}_{2} \cos \tilde{\beta}_{2}=\frac{\tilde{\xi}_{3} \tilde{K}_{23}-\tan \tilde{\beta}_{3} \tilde{\xi}_{2}}{\operatorname{det}\left(\tan \tilde{\beta}+\tilde{\kappa}^{c c}\right)}, \\
& \tilde{Z}_{3} \cos \tilde{\beta}_{3}=\frac{\tilde{\xi}_{2} \tilde{K}_{23}-\tan \tilde{\beta}_{2} \tilde{\xi}_{3}}{\operatorname{det}\left(\tan \tilde{\beta}+\tilde{\kappa}^{c c}\right),}
\end{aligned}
$$

where $\tilde{\xi}_{i}$ denotes $\tilde{K}_{1 i}^{o c}$ and $\tilde{\beta}_{i}$ denotes $\pi\left(v_{i}+\mu_{i}\right)$. The phase shift $\tilde{\delta}_{r}$, which is the phase of $\operatorname{det}\left(\tan \tilde{\beta}+\tilde{\kappa}^{c c}\right)$, satisfies

$$
\tan \tilde{\delta}_{r}=-\frac{\tilde{\xi}_{2}^{2} \tan \tilde{\beta}_{3}+\tilde{\xi}_{3}^{2} \tan \tilde{\beta}_{2}-2 \tilde{\xi}_{2} \tilde{\xi}_{3} \tilde{K}_{23}}{\tan \tilde{\beta}_{2} \tan \tilde{\beta}_{3}-\tilde{K}_{23}^{2}}
$$

The square of the modulus of $\operatorname{det}\left(\tan \tilde{\beta}+\tilde{\kappa}^{c c}\right)$ can be greatly simplified as

$$
C^{2}=\tilde{\xi}_{3}^{4} \tilde{W}_{2 e f f}^{2}\left(\tilde{\varepsilon}_{3}^{2}+1\right)\left(\tilde{\varepsilon}_{2 e f f}^{2}+1\right),
$$

where $\tilde{\varepsilon}_{i}$ denotes $\tan \tilde{\beta}_{i} / \tilde{\xi}_{i}^{2}(i=2,3)$. The effective parameters for Rydberg series 2 used in (8) are defined as follows:

$$
\tilde{\varepsilon}_{2 e f f}=\frac{\tan \tilde{\beta}_{2}}{\tilde{W}_{2 e f f}}-\tilde{S}
$$

$$
\tilde{W}_{2 e f f}=\tilde{W}_{2} \frac{\left(\tilde{\varepsilon}_{3}+\tilde{q}_{W}\right)^{2}}{\tilde{\varepsilon}_{3}^{2}+1},
$$

where $\tilde{W}_{i}$, denoting $\tilde{\xi}_{i}^{2}$, is the spectral width for the lines of Rydberg series $i$ and $\tilde{q}_{W}$, serving as a profile index for $\tilde{W}_{2 e f f}$, denotes

$$
\tilde{q}_{W}=-\frac{\tilde{K}_{23}}{\tilde{\xi}_{2} \tilde{\xi}_{3}}
$$

$\tilde{S}$ in Eq. (9) can be interpreted as a shift in resonance position, albeit inappropriate, and denotes

$$
\tilde{S}=\frac{\tilde{W}_{2}}{\tilde{W}_{2 e f f}} \frac{\left(\tilde{q}_{W}^{2}-1\right) \tilde{\varepsilon}_{3}-2 \tilde{q}_{W}}{\tilde{\varepsilon}_{3}^{2}+1} .
$$

$\tilde{W}_{2 \text { eff }}$ are the spectral widths of the lines of perturbed Rydberg series 2 and are the same as $\pi \Gamma v_{2}^{3} / 2$ considered extensively by Cooke and Cromer ${ }^{11}$ and Lecomte. ${ }^{13}$ The photoionization cross section can be reformulated as follows:

$$
\begin{aligned}
\sigma & =\tilde{\sigma}_{0} \frac{\left(\tilde{\varepsilon}_{3}+\tilde{q}_{3}\right)^{2}}{\tilde{\varepsilon}_{3}^{2}+1} \frac{\left(\tilde{\varepsilon}_{2 e f f}+\tilde{q}_{2 e f f}\right)^{2}}{\tilde{\varepsilon}_{2 e f f}^{2}+1} \\
& =\tilde{\sigma}_{0 I} \frac{\left(\tilde{\varepsilon}_{2 e f f}+\tilde{q}_{2 e f f}\right)^{2}}{\tilde{\varepsilon}_{2 e f f}^{2}+1}=\tilde{\sigma}_{0} \frac{\left(\tilde{\varepsilon}_{a}+\tilde{q}_{a}\right)^{2}}{\tilde{\varepsilon}_{a}^{2}+1},
\end{aligned}
$$

where $\tilde{q}_{i}$ is defined by $-D_{1} / \tilde{\xi}_{i} D_{i}(i=2,3)$ with the transition dipole moments $\tilde{D}_{i}$ to the $i$ th channel and $\tilde{\sigma}_{0 I}$ denotes the autoionization cross section of an isolated Rydberg series 3 acting as an interloper and forms the envelope:

$$
\tilde{\sigma}_{0 I}=\tilde{\sigma}_{0}\left(\tilde{\varepsilon}_{3}+\tilde{q}_{3}\right)^{2} /\left(\tilde{\varepsilon}_{3}^{2}+1\right)
$$

where $\tilde{\sigma}_{0}=\left(4 \pi^{2} \alpha \omega / 3\right) \tilde{D}_{1}^{2}$. It also serves as the "structured" continuum (or background) cross section. The line profile index $\tilde{q}_{2 e f f}$ for the lines of the perturbed Rydberg series 2 is defined by

$$
\tilde{q}_{2 e f f}=\tilde{S}+\frac{\tilde{W}_{2}}{\tilde{W}_{2 e f f}}\left[\tilde{q}_{2}-\frac{\left(\tilde{q}_{W}-\tilde{q}_{2}\right)\left(\tilde{q}_{W}-\tilde{q}_{3}\right)}{\tilde{\varepsilon}_{3}+\tilde{q}_{3}}\right] .
$$

Note that $\tilde{\sigma}_{0 I}$ is zero at $\tilde{\varepsilon}_{3}=-\tilde{q}_{3}$ from which we might wrongly conclude that $\sigma$ is zero there. But the cross section $\sigma$ is not zero but instead has a value of

$$
\begin{aligned}
\sigma= & \tilde{\sigma}_{0} \frac{\left[\left(\tilde{q}_{2}-\tilde{q}_{W}\right)\left(\tilde{q}_{3}-\tilde{q}_{W}\right)\right]^{2}}{\left(\tilde{\varepsilon}_{2}-\tilde{q}_{3}+2 \tilde{q}_{W}\right)^{2}+\left(\tilde{\varepsilon}_{2} \tilde{q}_{3}+\tilde{q}_{W}^{2}\right)^{2}}, \\
& \left(\text { when } \tilde{\varepsilon}_{3}=-\tilde{q}_{3}\right)
\end{aligned}
$$

because of the singularity of $\tilde{q}_{2 e f f}$ there according to Eq. (15). The last equality of Eq. (13) is due to Friedrich and Wintgen ${ }^{16}$ where $\tilde{\varepsilon}_{a}$ and $\tilde{q}_{a}$ are defined as 


$$
\begin{aligned}
& \tilde{\varepsilon}_{a}=\frac{\tilde{\varepsilon}_{2} \tilde{\varepsilon}_{3}-\tilde{q}_{W}^{2}}{\tilde{\varepsilon}_{2}+\tilde{\varepsilon}_{3}+2 \tilde{q}_{W}}, \\
& \tilde{q}_{a}=\frac{\left(\tilde{\varepsilon}_{2}+\tilde{q}_{W}\right) \tilde{q}_{3}+\left(\tilde{\varepsilon}_{3}+\tilde{q}_{W}\right) \tilde{q}_{2}}{\tilde{\varepsilon}_{2}+\tilde{\varepsilon}_{3}+2 \tilde{q}_{W}} .
\end{aligned}
$$

Although the last form in Eq. (13) is symmetric with respect to the two Rydberg series and are unsuitable for discussing the perturbed Rydberg series, it is particularly suitable to consider the phenomena around $\tan \tilde{\beta}_{2} \tan \tilde{\beta}_{3}=\tilde{K}_{23}^{2}$ or equivalently $\tilde{\varepsilon}_{2} \tilde{\varepsilon}_{3}=\tilde{q}_{W}^{2}$. The latter was the focal point in several studies ${ }^{15-16}$ because it approximates the resonance positions in the presence of an interloper. At the energies satisfying it, the autoionization cross section can be given simply by $\tilde{\sigma}=\tilde{\sigma}_{0} \tilde{q}_{a}^{2}$.

Further developments and applications of Ueda's formula. The complex resonance, where a dense Rydberg series is perturbed by one level of a coarse Rydberg series, can be viewed as a Rydberg series that interacts with the structured continuum from the point of view of the dense Rydberg series, or a coarse series chopped by the dense series from the point of view of the coarse one. Ueda developed a formulation that is best fitted to explain this phenomenon. Unfortunately, his formulation has not been used thus far. Other people took an approach that is valid only at the energies of bound states of the systems, where only two closed channels are included. Despite being limited to a particular energy, they yielded rather successful results but took more complicated forms.

Let us first consider the widths of the lines of the dense Rydberg series when the perturber is present. These widths can be obtained in Ueda's formula by expanding $\tilde{\varepsilon}_{2 e f f}$ into the Taylor series around the resonance center, i.e., $E \approx E_{R}$ or $\tan \tilde{\beta}_{2} \approx 0$, of series 2 with respect to energy $E$. From Eq. (9), one obtains

$$
\varepsilon_{2 e f f}=\left.\frac{d}{d E}\left(\frac{\tan \tilde{\beta}_{2}}{\tilde{W}_{2 e f f}}\right)\right|_{E_{R}}\left(E-E_{R}\right) \approx \frac{\pi}{2 \operatorname{Ryd} \tilde{W}_{2 e f f}\left(E_{R}\right)} v_{2}^{3}\left(E-E_{R}\right)
$$

where $d \tilde{\beta}_{2} / d E=\pi d v_{2} / d E=\pi v_{2}^{3} / 2$ Ryd is used. $\tilde{W}_{2 e f f}\left(E_{R}\right)$ denotes the value of $\tilde{W}_{2 e f f}$ at $E=E_{R}$ and Ryd denotes the Rydberg constant. The resonance peak width $\tilde{\Gamma}_{2 e f f}$ defined by $\tilde{\varepsilon}_{2 e f f}=2\left(E-E_{R}\right) / \tilde{\Gamma}_{2 \text { eff }}$ can be obtained as follows

$$
\tilde{\Gamma}_{2 e f f}=\frac{4 \operatorname{Ryd}}{\pi v_{2}^{3}} \tilde{W}_{2 e f f}\left(E_{R}\right)=\frac{4 \operatorname{Ryd}}{\pi v_{2}^{3}} \tilde{W}_{2} \frac{\left(\tilde{\varepsilon}_{3}+\tilde{q}_{W}\right)^{2}}{\tilde{\varepsilon}_{3}^{2}+1},
$$

where all the quantities are calculated at $E=E_{R}$. Wintgen and Friedrich ${ }^{16}$ obtained the corresponding width $\Gamma_{W F}$ using Smith's time delay ${ }^{19} \tau\left(=2 \hbar d \tilde{\delta}_{r} / d E\right)$ in addition to the relation $\Gamma=4 \hbar / \tau$ at the resonance energies satisfying $\tan \tilde{\beta}_{2}\left(E_{R}\right) \tan \tilde{\beta}_{3}\left(E_{R}\right)=\tilde{K}_{23}^{2}$ :

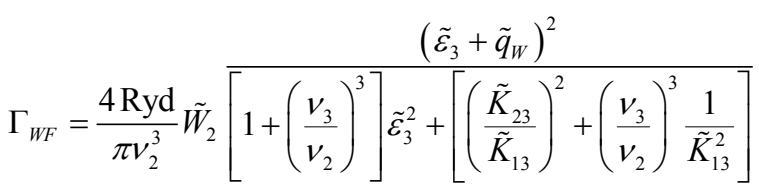

However, $\Gamma_{W F}$ cannot be compared with $\tilde{\Gamma}_{2 e f f}$ of Eq. (19) because it corresponds to the measured width of the resonance peaks as a whole while $\tilde{\Gamma}_{2 \text { eff }}$ only shows the width of the perturbed Rydberg series with perturber's contribution subtracted.

It may be desirable to express the entire width $\Gamma$ in terms of $\tilde{\Gamma}_{2 e f f}$ because $\tilde{\Gamma}_{2 e f f}$ is the disentangled entity with a clear physical origin. Since time delays and widths can be related using a simple relation, let us consider the time delay instead of the width. The precise formula of the time delay can be obtained easily as follows:

$\tau_{D}=2 \hbar$
$\frac{\left(1+\tan ^{2} \tilde{\beta}_{2}\right)\left(\tilde{\xi}_{3} \tilde{K}_{23}-\tilde{\xi}_{2} \tan \tilde{\beta}_{3}\right)^{2} \frac{d \tilde{\beta}_{2}}{d E}+\left(1+\tan ^{2} \tilde{\beta}_{3}\right)\left(\tilde{\xi}_{2} \tilde{K}_{23}-\tilde{\xi}_{3} \tan \tilde{\beta}_{2}\right)^{2} \frac{d \tilde{\beta}_{3}}{d E}}{\left(\tan \tilde{\beta}_{2} \tan \tilde{\beta}_{3}-\tilde{K}_{23}^{2}\right)^{2}+\left(\tilde{\xi}_{2}^{2} \tan \tilde{\beta}_{3}+\tilde{\xi}_{3}^{2} \tan \tilde{\beta}_{2}-2 \tilde{\xi}_{2} \tilde{\xi}_{3} \tilde{K}_{23}\right)^{2}}$.

Normally, the second term in the numerator can be ignored because the energy variation of $\tilde{\beta}_{3}$ is much smaller than that of $\tilde{\beta}_{2}$. Utilizing both this and the simplified form of (8) of the denominator, the above equation can be written as follows:

$$
\tau_{D} \cong \tau_{D 2}^{\text {isolated }} \frac{\tilde{W}_{2}}{\tilde{W}_{2 e f f}} \frac{\tilde{\varepsilon}_{2}^{2}+1}{\tilde{\varepsilon}_{2 e f f}^{2}+1}
$$

where $\tau_{D 2}^{\text {isolated }}$ is the time delay at the lines of the Rydberg series 2 when there is no interloper and is given by

$$
\tau_{D 2}^{\text {isolated }}=\frac{\pi \hbar}{\mathrm{Ryd}} \tilde{W}_{2} v_{2}^{3} \frac{\tilde{\varepsilon}_{2}^{2}+W_{2}^{-2}}{\tilde{\varepsilon}_{2}^{2}+1} .
$$

The width function $\Gamma$ may be defined at all energies by $\Gamma=4 \hbar / \tau_{D}$. Eq. (21) yields the intended disentangled relation

$$
\Gamma=\tilde{\Gamma}_{2 e f f} \frac{1+\tilde{\varepsilon}_{2 e f f}^{2}}{1+\tilde{\varepsilon}_{2}^{2} \tilde{W}_{2}^{2}},
$$

where $\tilde{\Gamma}_{2 \text { eff }}$ has the same form as (19) but defined for all energies:

$$
\tilde{\Gamma}_{2 e f f}=\frac{4 \operatorname{Ryd}}{\pi v_{2}^{3}} \tilde{W}_{2} \frac{\left(\tilde{\varepsilon}_{3}+\tilde{q}_{W}\right)^{2}}{\tilde{\varepsilon}_{3}^{2}+1}=\frac{4 \mathrm{Ryd}}{\pi v_{2}^{3}} \tilde{W}_{2 e f f} .
$$

$\tilde{W}_{2 e f f}$ is already defined in (10). Note that the width function is zero at $\tilde{\varepsilon}_{3}=-\tilde{q}_{W}$ as $\tilde{\Gamma}_{2 e f f}$ is zero there.

Eq. (21) shows the interesting dynamics in that the interloper Rydberg series 3 affects the time delay only indirectly. Hence, the behavior of the time delay is dominated by the dense series 2. This is in contrast to the autoionization cross section (13) whose overall behavior is governed by the interloper's one. This difference might be understood if one recalls that the absorption process is instant and the dominant absorption process is determined by the magnitude of the transition dipole moments, which is the transition to the interloper line, because the smaller size of its wavefunction resembles the ground wavefunction more closely and greater overlap is obtained. Since the residence time 
or orbiting period is proportional to $v^{3}$, it is short for the lower line of the Rydberg series, which is the case for an interloper. This suggests that an excited electron quickly leaks into the dense series, which has a much longer orbiting period of time.

Let us consider as another example of the difference between the results from different approaches ${ }^{15}$ for the maximum intensity of the dense Rydberg series, whose connected line forms an envelope. This was obtained in Ueda's formula by inserting $\tilde{\varepsilon}_{2 e f f}=1 / \tilde{q}_{2 e f f}$ as

$$
\sigma_{\max }=\tilde{\sigma}_{0} \frac{\left(\tilde{\varepsilon}_{3}+\tilde{q}_{3}\right)^{2}}{\tilde{\varepsilon}_{3}^{2}+1}\left(\tilde{q}_{2 e f f}^{2}+1\right)
$$

and approximated in Giusti-Suzor and Lefebvre-Brion's ${ }^{15}$ at the resonance energies satisfying $\tan \tilde{\beta}_{2}\left(E_{R}\right) \tan \tilde{\beta}_{3}\left(E_{R}\right)=\tilde{K}_{23}^{2}$, or $\tilde{\varepsilon}_{2} \tilde{\varepsilon}_{3}=\tilde{q}_{W}^{2}$, as follows :

$$
\sigma_{\max }=\tilde{\sigma}_{0}\left(\frac{\tilde{\varepsilon}_{3}+\tilde{q}_{3} \tilde{q}_{W} / \tilde{q}_{2}}{\tilde{\varepsilon}_{3}+\tilde{q}_{W}}\right)^{2} \tilde{q}_{2}^{2},\left(\text { when } \tilde{K}_{23} \neq 0\right) .
$$

Eq. (26) becomes Eq. (25) when $\tilde{q}_{\text {2eff }}$ is dominated by the pole at $\tilde{\varepsilon}_{3}=-\tilde{q}_{W}$ as well as when $\tilde{q}_{3} \gg \tilde{q}_{2}, \tilde{q}_{W}$ are satisfied, where $\tilde{q}_{2 e f f}$ is $-\left(1+\tilde{q}_{W}^{2}\right) /\left(\tilde{\varepsilon}_{3}+\tilde{q}_{W}\right)$. Such conditions are usually met. The shift and width of the resonance peak of a perturber were found to be equal to those of the unperturbed one. A cursory look at the spectra contradicts this. The spectra were considered to be undergoing a great change when the mutual interaction between the closed channels is introduced. It will be shown later that this apparent great change has another origin. The different conclusion drawn in previous work ${ }^{15}$ is due to the inability to see each spectrum of the envelope and dense Rydberg series separately. Note that intensity enhancement can only be seen in the dense Rydberg series and amounts to $\tilde{q}_{2 \text { eff }}^{2}+1$. No enhancement could be seen in the envelope. (Because there is no theory for the coupling between an envelope and perturbed spectra of dense series, it is unclear whether the lack of enhancement in the envelope was instituted intentionally from the outset.)

Pole structures in parameters. Since the behavior of dynamic parameters can be greatly influenced by the poles, let us examine their pole structure. In particular, the resonance positions are intimately related to the poles of $\tilde{\varepsilon}_{2 e f f}$ and the $q$-reversals are related to those of $\tilde{q}_{2 e f f}$. For this, we need a decomposition of $\tilde{S}$ in Eq. (12) into partial fractions, ${ }^{20}$ which contained only the principal part with the second order pole located at $\tilde{\varepsilon}_{3}=-\tilde{q}_{W}$ as follows:

$$
\tilde{S}=\frac{\tilde{q}_{W}^{2}-1}{\tilde{\varepsilon}_{3}+\tilde{q}_{W}}-\frac{\tilde{q}_{W}\left(1+\tilde{q}_{W}^{2}\right)}{\left(\tilde{\varepsilon}_{3}+\tilde{q}_{W}\right)^{2}} .
$$

Although the pole of second order exists in $\tilde{S}$, it cancels out and only the first order pole remains in the decomposition of $\tilde{q}_{\text {2eff }}$ in Eq. (15) into partial fractions as follows:

$$
\tilde{q}_{2 e f f}=\tilde{q}_{2}-\left(1+\tilde{q}_{3}^{2}\right) \frac{\tilde{q}_{2}-\tilde{q}_{W}}{\tilde{q}_{3}-\tilde{q}_{W}} \frac{1}{\tilde{\varepsilon}_{3}+\tilde{q}_{3}}-\left(1+\tilde{q}_{W}^{2}\right) \frac{\tilde{q}_{2}-\tilde{q}_{3}}{\tilde{q}_{W}-\tilde{q}_{3}} \frac{1}{\tilde{\varepsilon}_{3}+\tilde{q}_{W}},
$$

which is composed of the entire part $\tilde{q}_{2}$ and the principal part with simple poles at $-\tilde{q}_{3}$ and $-\tilde{q}_{W}$. This shows that the interloper to the line profile index $\tilde{q}_{2 \text { eff }}$ for the autoionization cross section of Rydberg series 2 enters as simple poles at $\tilde{\varepsilon}_{3}=-\tilde{q}_{3}$ and $-\tilde{q}_{W}$.

For the width, the effect of an interloper enters as simple poles not on the real axis but on the imaginary axis at $\pm i$ :

$$
\tilde{W}_{2 e f f}=\tilde{W}_{2}-\frac{i}{2} \tilde{W}_{2}\left[\frac{\left(\tilde{q}_{W}+i\right)^{2}}{\tilde{\varepsilon}_{3}-i}-\frac{\left(\tilde{q}_{W}-i\right)^{2}}{\tilde{\varepsilon}_{3}+i}\right] .
$$

Since the poles do not lie on the real axis, there is no singular behavior on the real axis, and $\tilde{W}_{2 \text { eff }}$ is always bounded (finite channel-channel coupling strengths).

Finally, the decomposition of $\tilde{\varepsilon}_{2 e f f}$ into partial fractions can be obtained as follows:

$$
\tilde{\varepsilon}_{2 e f f}=\tilde{\varepsilon}_{2}-\frac{2 \tilde{q}_{W}}{\tilde{\varepsilon}_{3}+\tilde{q}_{W}}\left(\tilde{\varepsilon}_{2}+\frac{\tilde{q}_{W}^{2}-1}{2 \tilde{q}_{W}}\right)+\left(1+\tilde{q}_{W}^{2}\right) \frac{\tilde{\varepsilon}_{2}+\tilde{q}_{W}}{\left(\tilde{\varepsilon}_{3}+\tilde{q}_{W}\right)^{2}} .
$$

The perturbation of the reduced energy parameter $\tilde{\varepsilon}_{2 e f f}$ from $\tilde{\varepsilon}_{2}$ by an interloper enters as the principal part with the second order pole at $\tilde{\varepsilon}_{3}=-\tilde{q}_{W}$. At the pole, $\tilde{\varepsilon}_{2 e f f}$ exhibits complicated behavior but the number of cycles is unaffected by it. Otherwise, its behavior would be similar to $\tilde{\varepsilon}_{2}$ and shows a regular periodic pattern. Since Rydberg series 3 is further from the ionization threshold than Rydberg series $2, \tilde{\varepsilon}_{3}$ is mostly zero and can be ignored in Eq. (30). If $\tilde{q}_{W} \gg 1, \tilde{q}_{W}^{2}+1 \approx \tilde{q}_{W}^{2}$ and $\tilde{\varepsilon}_{2 e f f}$ in Eq. (30) are almost zero, which can explain the flat behavior of $\tilde{\varepsilon}_{2 e f f}$ compared to $\tilde{\varepsilon}_{2}$.

The fact that the order of pole of $\tilde{\varepsilon}_{2 e f f}$ is second has an unexpected consequence that the huge enhancement $\tilde{q}_{2 e f f}^{2}+1$ occurring due to that pole is completely suppressed at the very pole since $\tilde{q}_{2 e f f}$ is dominated by $\tilde{\varepsilon}_{2 e f f}$ near the pole and can be ignored so that the enhancement factor is 1 instead of $\tilde{q}_{2 e f f}^{2}+1$. In most case, this suppression can be ignored if the resonance peak of the dense series does not lie near the pole. No peaks at $v_{2}=17$ in the spectrum of (b) $\tilde{K}_{13}=0.1 \pi$ of Fig. $5, v_{2}=18$ in (b) $\tilde{K}_{13}=$ $0.12 \pi$ and $v_{2}=19$ in (d) $\tilde{K}_{13}=0.15 \pi$ of Fig. 9 are due to this suppression.

$q$ Reversals. The spectral shape of autoionization cross sections undergoes a conspicuous change when the line profile asymmetric index $q$ undergoes a sign change. It changes sign either at the zero or at the simple pole. It should be noted that $\tilde{q}_{2 \text { eff }}$ is a simple pole so that the sign is reversed. The sign would remain the same if the pole was of a second order. It was already found that the poles of $\tilde{q}_{2 e f f}$ are at $-\tilde{q}_{3}$ and $-\tilde{q}_{W}$. Eq. (28) shows that the zeros of $\tilde{q}_{2 e f f}$, another source of $q$ reversal, occur mainly at two values of $\tilde{\varepsilon}_{3}$, which are the roots of the quadratic equation $\tilde{q}_{2 e f f}\left(\tilde{\varepsilon}_{3}\right)=0$ :

$$
\tilde{q}_{2} \tilde{\varepsilon}_{3}^{2}-\left(1-\tilde{q}_{3} \tilde{q}_{W}\right) \tilde{\varepsilon}_{3}+\tilde{q}_{2}-\tilde{q}_{3}-\tilde{q}_{W}=0 .
$$

The real roots of $\tilde{q}_{2 e f f}=0$ exist when the discriminant of the quadratic equation ( 31 ) is $\geq$ zero: 


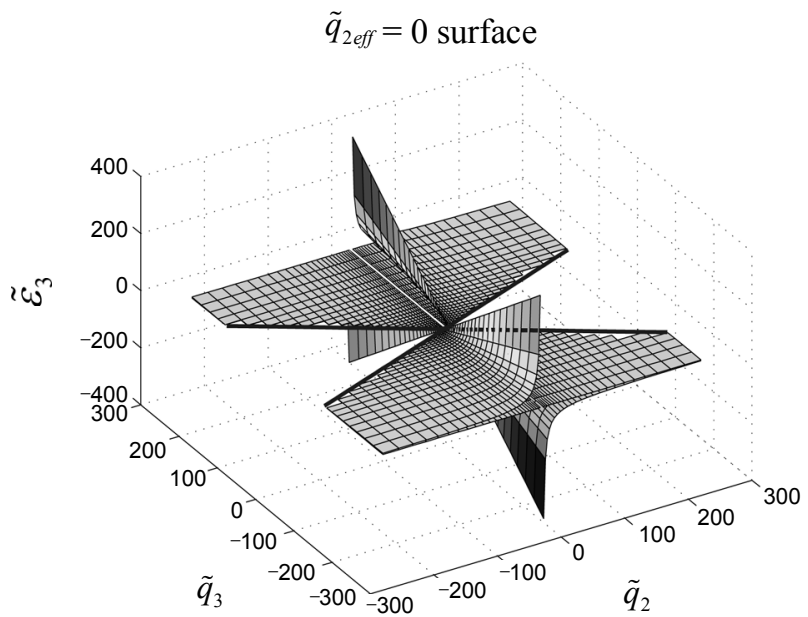

Figure 1. $\tilde{q}_{2 e f f}=0$ surfaces for $\tilde{q}_{W}=-4$. The curves for the double root are also shown with heavy lines.

$$
D=\left(1-\tilde{q}_{3} \tilde{q}_{W}\right)^{2}+4 \tilde{q}_{2}\left(\tilde{q}_{3}+\tilde{q}_{W}-\tilde{q}_{2}\right) \geq 0 .
$$

With $\tilde{q}_{W}$ fixed, the two roots of Eq. (31) form two root surfaces of $\tilde{\varepsilon}_{3 \text { root }}=\tilde{\varepsilon}_{3 \text { root }}\left(\tilde{q}_{2}, \tilde{q}_{3}\right)$, bounded by the double root curves, whose precise forms are easily obtained. However, in most cases, it is enough to have asymptotes that are given by $\left[\tilde{q}_{2}, \tilde{q}_{3}^{\mathrm{dr}}\right.$, $\left.\left(1-\tilde{q}_{3}^{\mathrm{dr}} \tilde{q}_{W}\right) /\left(2 \tilde{q}_{2}\right)\right]$, where $\tilde{q}_{3}^{\mathrm{dr}}$ denote $-2 \tilde{q}_{2}\left[1 \pm\left(1+\tilde{q}_{W}^{2}\right)^{1 / 2}\right] / \tilde{q}_{W}^{2}$. Fig. 1 shows the zero surfaces for $\tilde{q}_{W}=-4$. Note that one of the two root surfaces is almost a plane close to zero while another surface behaves like a hyperbola, with a singular point at $\tilde{q}_{2}=0$. To understand this behavior, consider $\tilde{q}_{2} \ll \tilde{q}_{3}$, where two roots of Eq. (31) can be approximated as follows:

$$
\tilde{\varepsilon}_{3} \sim \frac{1-\tilde{q}_{3}-\tilde{q}_{W}}{\tilde{q}_{2}},-\frac{\tilde{q}_{3}+\tilde{q}_{W}-\tilde{q}_{2}}{1-\tilde{q}_{3} \tilde{q}_{W}} \sim-\frac{\tilde{q}_{3}+\tilde{q}_{W}}{\tilde{q}_{2}}, \frac{1}{\tilde{q}_{3}}+\frac{1}{\tilde{q}_{W}},
$$

The second root of (33) is almost zero if $\tilde{q}_{3}$ and $\tilde{q}_{W}$ are $>>1$ while the first one behaves as a hyperbola along the $\tilde{q}_{2}$ axis.

Consider the case where the value of $\tilde{K}_{13}$ increases while other parameters are kept constant, which is frequently considered to study the effect of coupling strength on autoionization spectra. ${ }^{4}$ Note that change in the value of $\tilde{K}_{13}$ does not simply bring about a change in $\tilde{q}_{3}$ alone. It also brings about a change in the value of $\tilde{q}_{W}$. Therefore, knowing both $\tilde{q}_{2}$ and $\tilde{q}_{3}$ is not enough to determine the root surfaces of $\tilde{q}_{2 \text { eff }}=0$. The value of $\tilde{q}_{W}$ is also needed. This means that the different root surface corresponding to different $\tilde{q}_{W}$ should be used for each $\tilde{K}_{13}$ value. Fig. 7 shows the root surfaces for the $\tilde{K}_{13}$ values corresponding to the spectra of 4 different $\tilde{K}_{13}$ values in Fig. 3 . The cylinders are also shown at $\left(\tilde{q}_{2}, \tilde{q}_{3}\right)$ corresponding to each spectrum. $\tilde{q}_{3}$ and $\tilde{q}_{W}$ decrease in magnitude in the same proportion if the value of $\tilde{K}_{13}$ increases in magnitude. This means that the cylinder moves toward the zero of $\tilde{q}_{3}$ (or $\tilde{q}_{2}$ axis) along the axis parallel to $\tilde{q}_{3}$ axis. For $\left|\tilde{q}_{W}\right| \ll 1$, the asymptote of the double root of zero surfaces of $\tilde{q}_{2 e f f}$ becomes either $\tilde{q}_{3}=\tilde{q}_{2}$ or $\tilde{q}_{3}=-\left(4 / \tilde{q}_{W}^{2}\right)$ $\tilde{q}_{2}$. The latter is almost equal to $\tilde{q}_{3}$ axis because $\left|\tilde{q}_{W}\right| \ll 1$. This means that the region of the $\left(\tilde{q}_{2} \tilde{q}_{3}\right)$ plane bounded by the line $\tilde{q}_{3}=\tilde{q}_{2}$ and $\tilde{q}_{3}$ axis can have real roots of $\tilde{\varepsilon}_{3}$, which covers approximately half the entire plane of $\left(\tilde{q}_{2} \tilde{q}_{3}\right)$. When the coupling strength $\tilde{K}_{13}$ is quite weak, $\left|\tilde{q}_{W}\right| \gg 1$ and the asymptotes become $\tilde{q}_{3}= \pm\left(2 / \tilde{q}_{W}\right) \tilde{q}_{2}$, which means that the real roots are allowed on most of the entire $\left(\tilde{q}_{2} \tilde{q}_{3}\right)$ plane.

There are 20 possible patterns of $q$ reversals when the cases have no real roots, double real root and two different real roots are all counted, as shown in Fig. 2. In addition, one more pattern corresponding to the root of a linear equation is possible for $\tilde{q}_{2}=0$. Therefore, in total, 21 patterns are possible for $q$ reversals. It may be argued that this consideration is purely mathematical and should be born out in the real system in order to be accepted as a meaningful piece of work. But note that there are no limits on the real values to be taken by either the reactance matrix elements or the transition dipole moments which determine the 3 line profile indices and subsequently the $q$ reversal

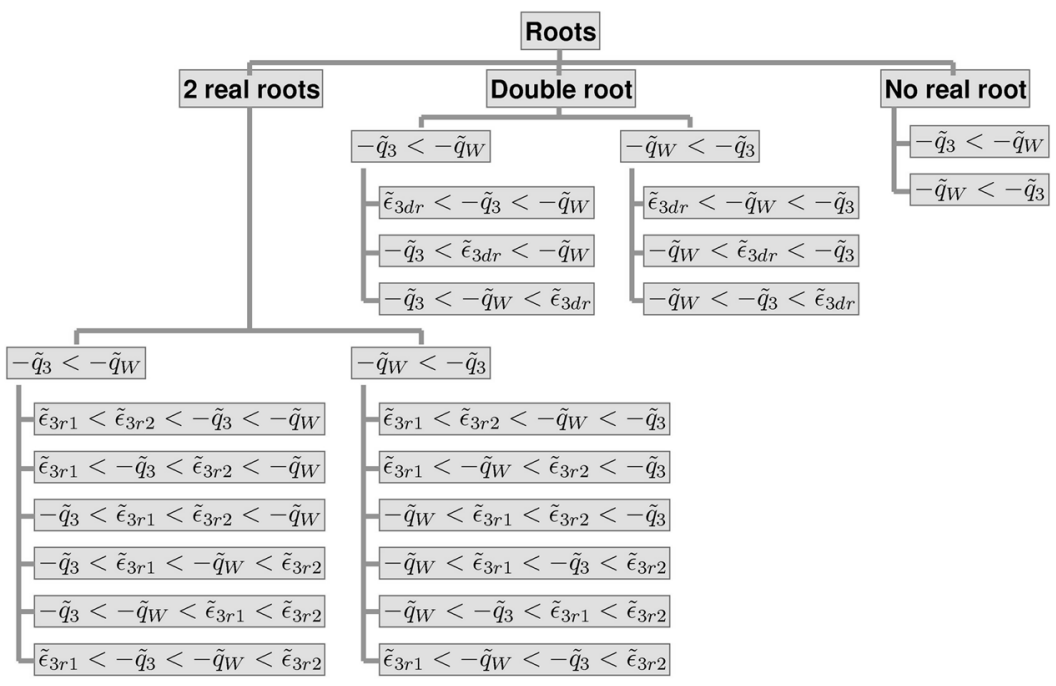

Figure 2. 20 possible patterns of $q$ reversals. In addition, one more pattern corresponding to the root of a linear equation is possible for $\tilde{q}_{2 e f f}=$ 0 . Therefore, in total, 21 patterns are possible for $q$ reversals. 


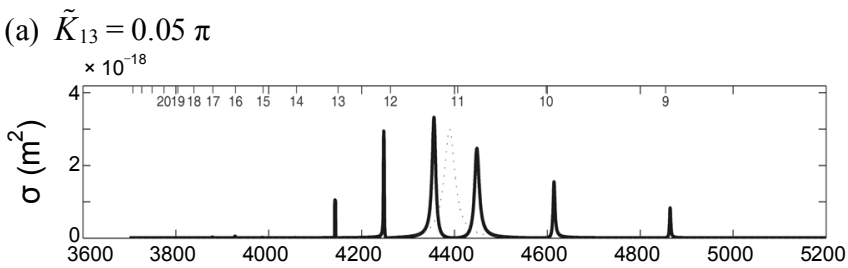

(b) $\tilde{K}_{13}=0.1 \pi$

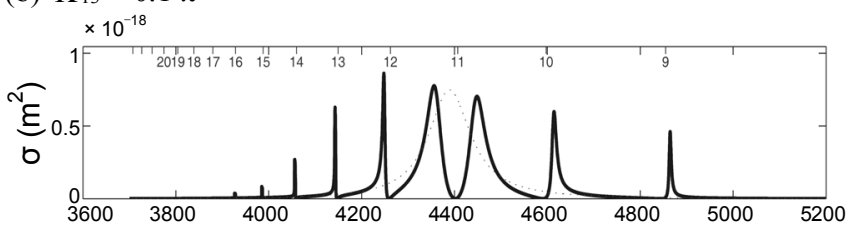

(c) $\tilde{K}_{13}=0.2 \pi$

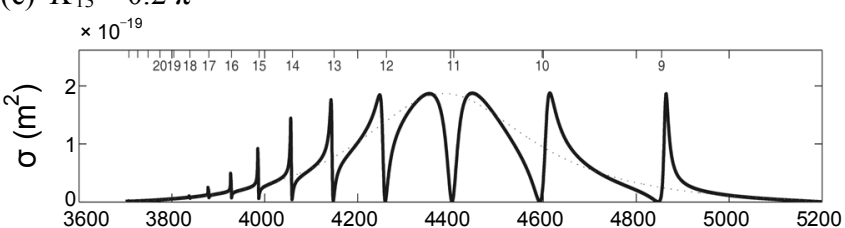

(d) $\tilde{K}_{13}=0.3 \pi$

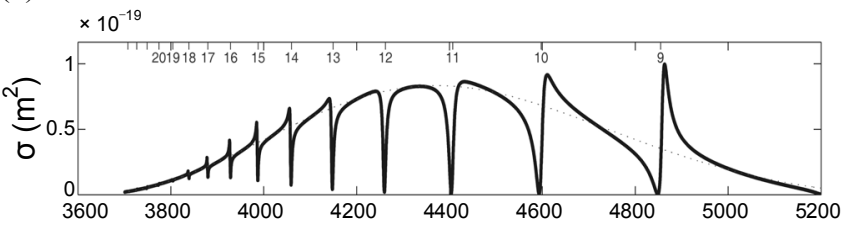

(e) $\tilde{K}_{13}=0.4 \pi$

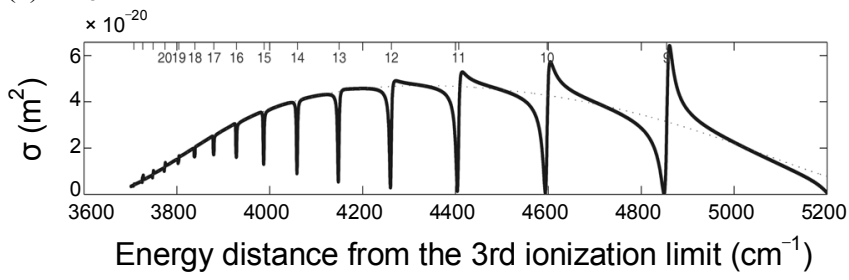

Figure 3. Effects of the widths of an interloper on dense Rydberg series. The smoothed spectra with instrumental half-width of $1 \mathrm{~cm}^{-1}$ are drawn with a heavy line. The spectra of the interloper itself are also shown with a dotted line. The first, second and third ionization threshold corresponding to channels 1,2 and 3 are taken as $0,6500,10000$ $\mathrm{cm}^{-1}$, respectively. The respective transition dipole moments are taken to be $1,18,150$ a.u.. $\tilde{K}_{12}=0.02 \pi, \tilde{K}_{23}=0.1 \pi$. $\tilde{K}_{13} / \pi=0.05,0.1,0.2,0.3$, 0.4 from the above. No phase renormalization was performed, i.e. $\mu_{i}=$ $0(i=1,2,3)$.

patterns. [For the channel wavefunctions in Eq. (3), transition dipole moments are real.]

Model studies of overlapping resonances. The most systematic studies on autoionization spectra due to an overlapping resonance were carried out by Lane, Connerade and colleagues. ${ }^{3}$ Most of their extensive studies of overlapping resonances in autoionization were performed by scattering calculations where the transition dipole moments were absent and could not be accepted seriously. Let us start by reproducing their interesting spectra showing a variety of aspects of overlapping resonances with the MQDT based on Ueda's ${ }^{17}$ formulation, which includes the transition dipole moments as a matter of fact.

Fig. 3 shows the effect of the width of an interloper on the

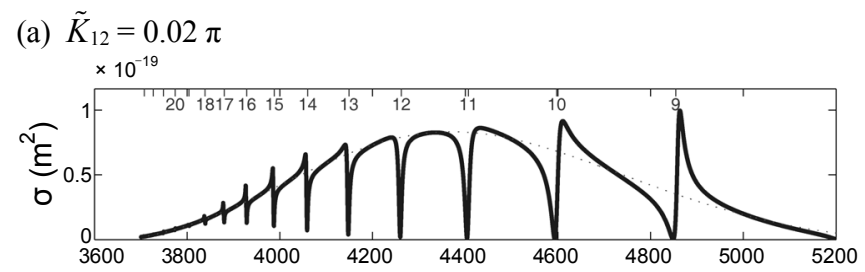

(b) $\tilde{K}_{12}=0.08 \pi$

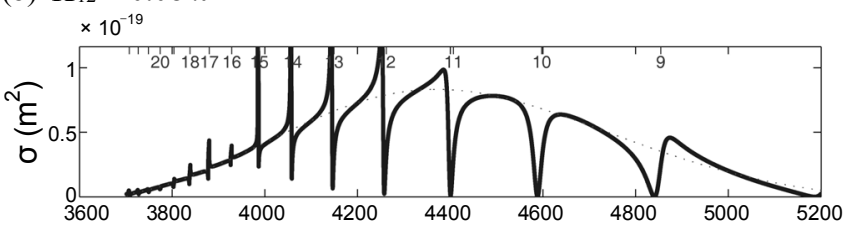

(c) $\tilde{K}_{12}=0.32 \pi$

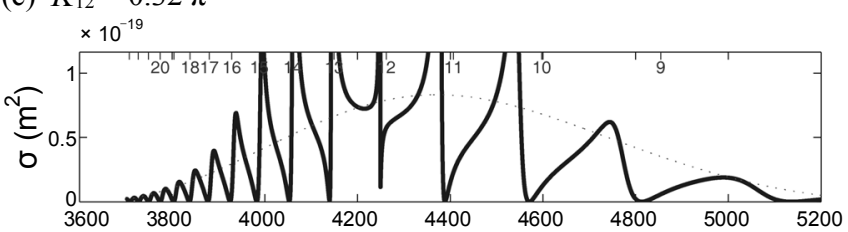

(d) $\tilde{K}_{12}=1.28 \pi$

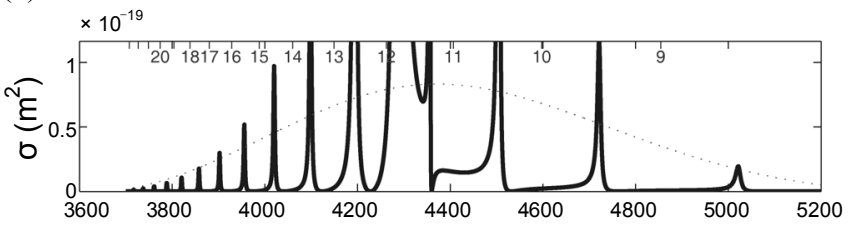

(e) $\tilde{K}_{12}=5.14 \pi$

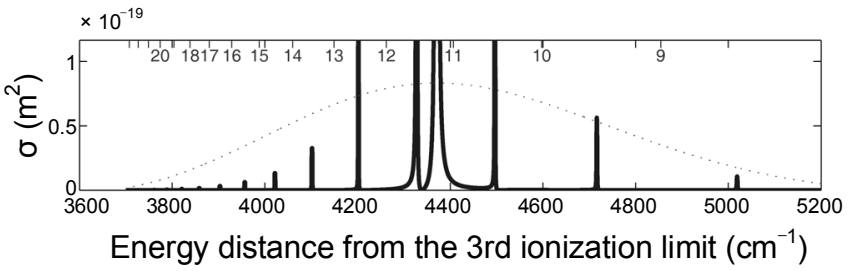

Figure 4. Effects of the widths of the dense Rydberg series on the line profiles and widths in its own overlapping resonance spectra. The first, second and third ionization threshold corresponding to channels 1,2 and 3 were taken as $0,6500,10000 \mathrm{~cm}^{-1}$, respectively. The respective transition dipole moments are taken to be $1,18,150$ a.u.. $\tilde{K}_{13}=0.3 \pi$, $\tilde{K}_{23}=0.1 \pi . \quad \tilde{K}_{12} / \pi=0.02,0.08,0.32,1.28,5.12$ from the above. No phase renormalization was performed, i.e. $\mu_{i}=0(i=1,2,3)$.

dense Rydberg series as $\tilde{K}_{13}$ is varied progressively from $0.05 \pi$, via $0.1 \pi, 0.2 \pi, 0.3 \pi$ to $0.4 \pi$. The interloper spectra alone are also included as dotted lines in the figure for comparison. The overall shapes of the spectra are the interloper spectra chopped by the lines of the dense perturbed series. Note that $q$ reversal occurs around the peak of the interloper so that peaks of the dense series are mirrored around the peak of the interloper. As the width of the interloper increases, the shapes of the lines undergo a large change. The lower graphs show the $q$ reversal straddling the intervening resonances extensively studied in Ref. [3-4]. It should be noted that the cross sections do not decrease to zero between successive autoionizing levels because of the instrumental smoothening with a bandwidth of $1 \mathrm{~cm}^{-1}$. 
(a) $\tilde{K}_{13}=0.05 \pi$
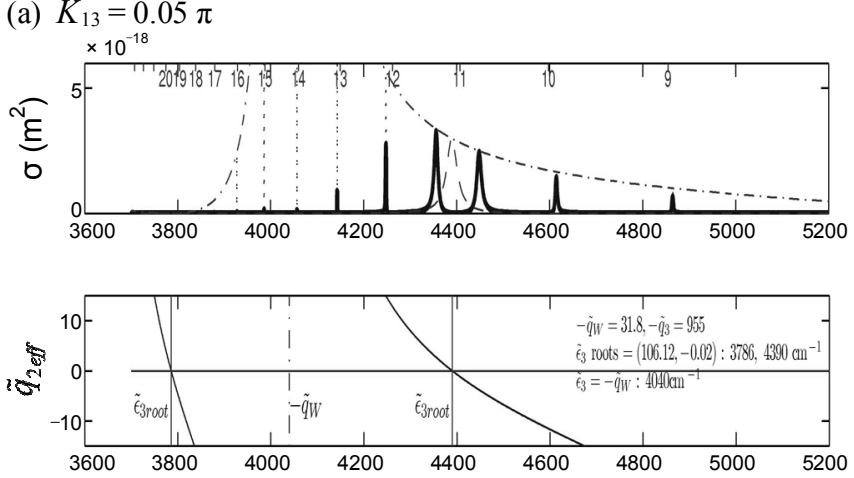

(b) $\tilde{K}_{13}=0.1 \pi$
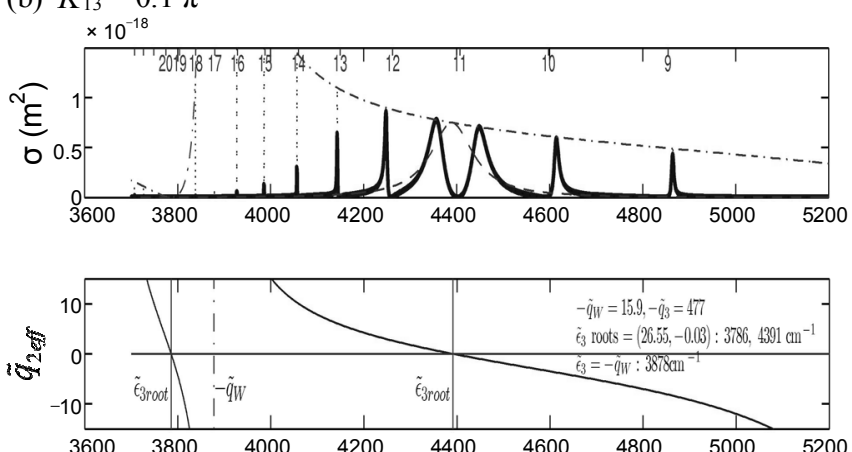

(c) $\tilde{K}_{13}=0.2 \pi$
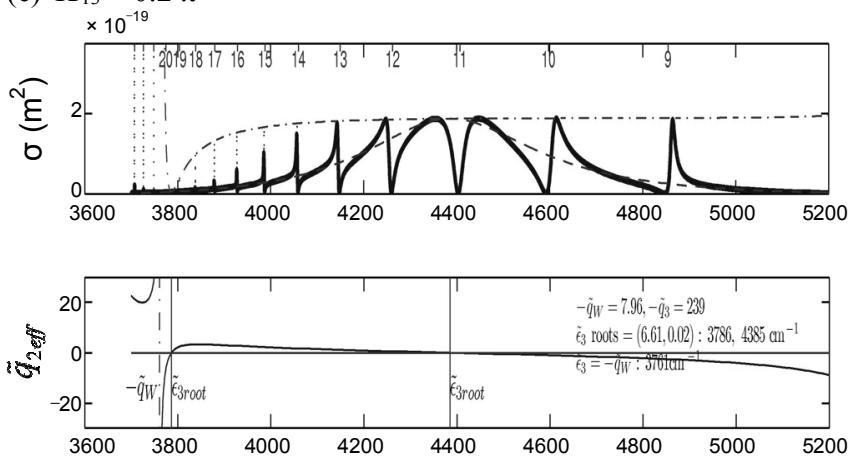

(d) $\tilde{K}_{13}=0.3 \pi$
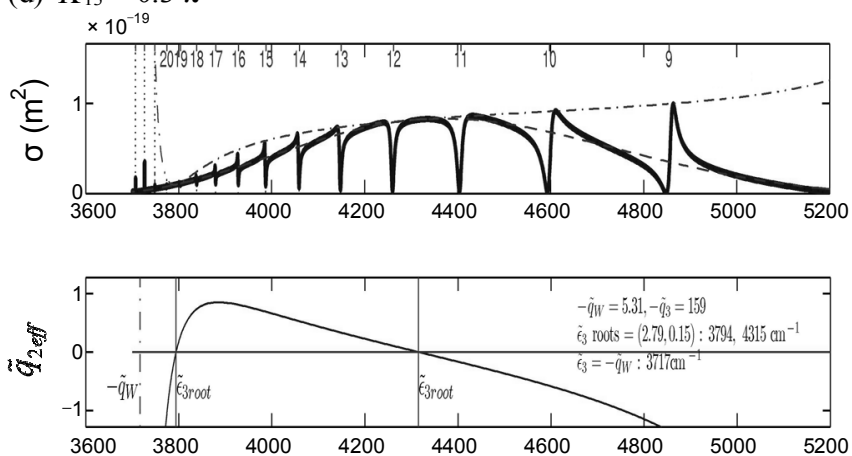

Energy distance from the 3rd ionization limit $\left(\mathrm{cm}^{-1}\right)$

Figure 5. $\tilde{q}_{2 e f f}$ graphs corresponding to Fig. 3 excluding the last graph. In autoionization spectra $\sigma$, the smoothed spectra with instrumental half-width of $1 \mathrm{~cm}^{-1}$ are drawn with a heavy line, the unsmoothed one with a dotted line, the envelope with a dashed line, $\sigma_{\max }$ with a dashdotted line. This convention is applied in other autoionization spectra, too. (a) $\tilde{K}_{12}=0.02 \pi$
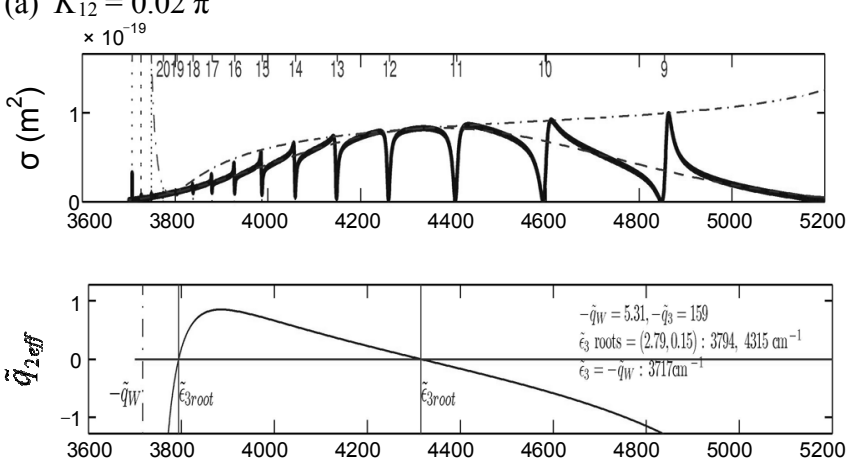

(b) $\tilde{K}_{12}=0.08 \pi$
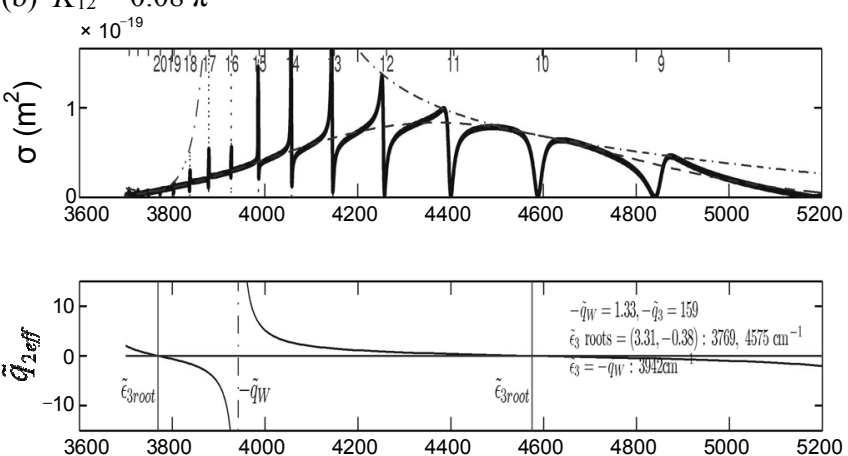

(c) $\tilde{K}_{12}=0.32 \pi$
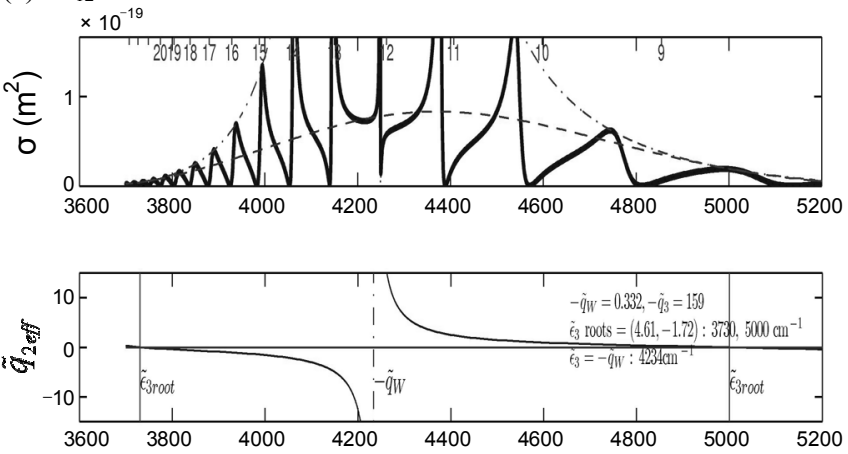

(d) $\tilde{K}_{12}=1.28 \pi$
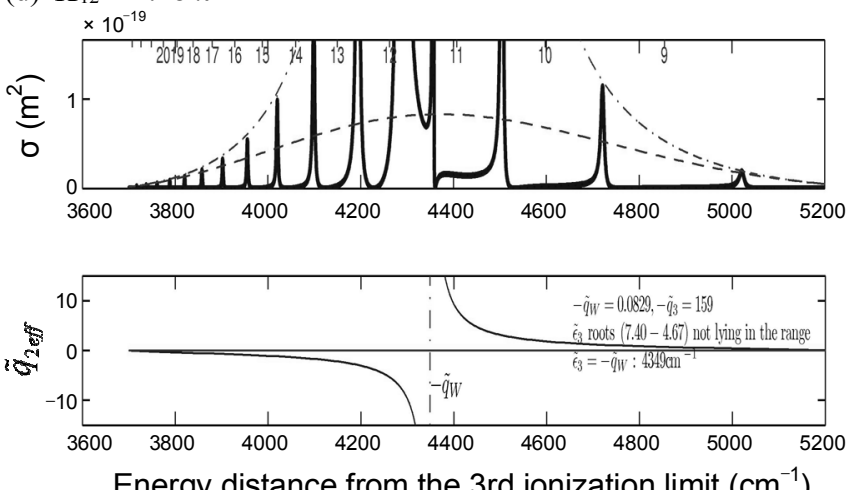

Figure 6. $\tilde{q}_{2 e f f}$ graphs corresponding to Fig. 4 excluding the last graph. 
(a) $\tilde{K}_{13}=0.05 \pi\left(\tilde{q}_{3}=-954.9, \tilde{q}_{W}=-31.8\right)$

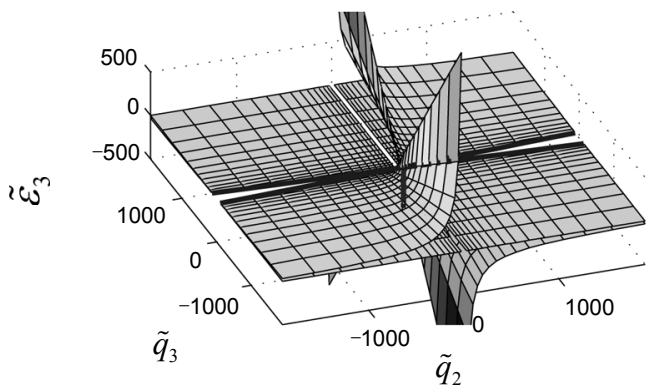

(c) $\tilde{K}_{13}=0.2 \pi\left(\tilde{q}_{3}=-238.7, \tilde{q}_{W}=-8.0\right)$

$2 \omega^{m}$

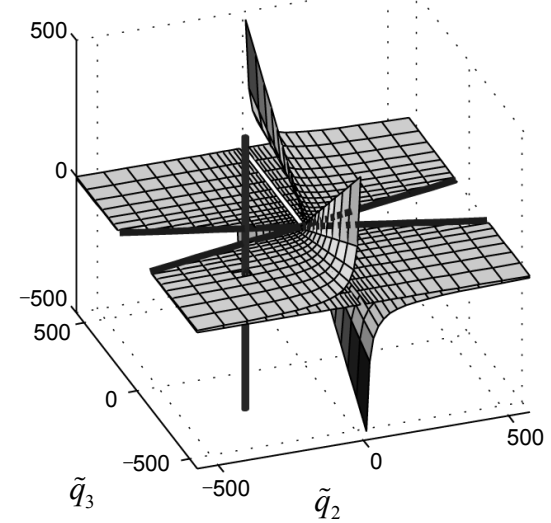

(b) $\tilde{K}_{13}=0.1 \pi\left(\tilde{q}_{3}=-477.5, \tilde{q}_{W}=-15.9\right)$

$1 \omega^{m}$

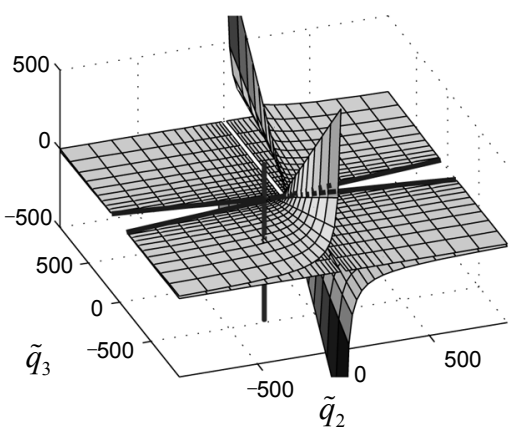

(d) $\tilde{K}_{13}=0.3 \pi\left(\tilde{q}_{3}=-159.2, \tilde{q}_{W}=-5.3\right)$

$2 \omega^{m}$

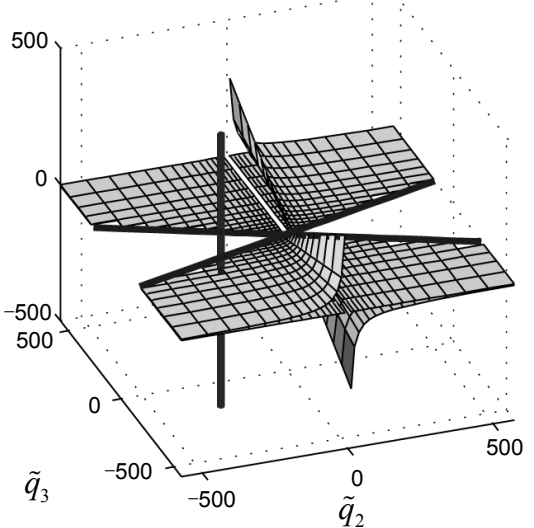

Figure 7. Zero surfaces of $\tilde{q}_{2 e f f}$ corresponding to Fig. 3 excluding the last graph. $\tilde{q}_{2}=-286.5$ for all cases.

(a) $\tilde{K}_{12}=0.02 \pi\left(\tilde{q}_{2}=-286.5, \tilde{q}_{W}=-5.3\right)$

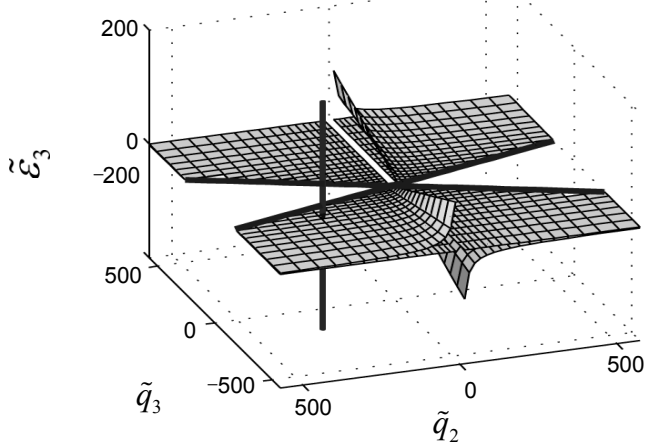

(c) $\tilde{K}_{12}=0.32 \pi\left(\tilde{q}_{2}=-35.8, \tilde{q}_{W}=-0.7\right)$

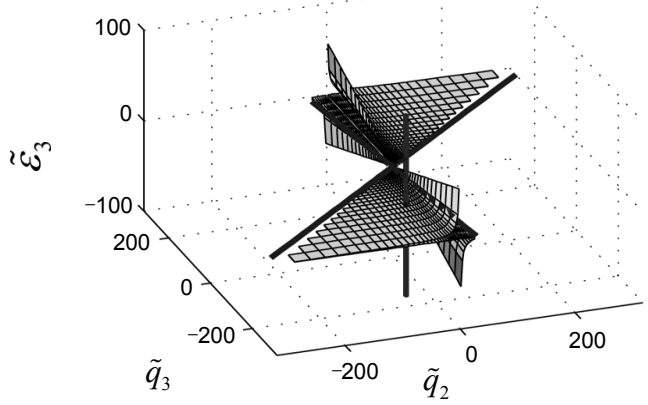

(b) $\tilde{K}_{12}=0.08 \pi\left(\tilde{q}_{2}=-71.6, \tilde{q}_{W}=-1.3\right)$

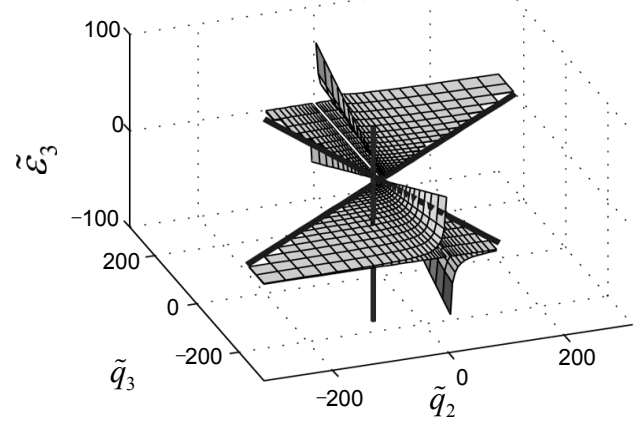

(d) $\tilde{K}_{12}=1.28 \pi\left(\tilde{q}_{2}=-17.9, \tilde{q}_{W}=-0.3\right)$

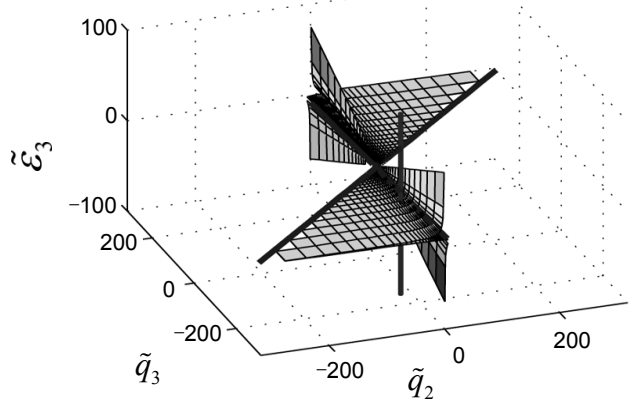

Figure 8. Zero surfaces of $\tilde{q}_{2 e f f}$ corresponding to Fig. 4 excluding the last graph. $\tilde{q}_{3}=-159.2$ for all cases. 
(a) $\tilde{K}_{13}=0.06 \pi$
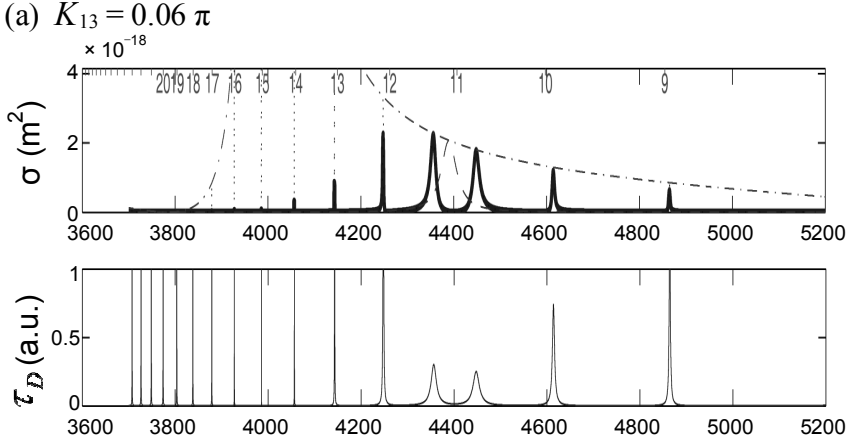

(c) $\tilde{K}_{13}=0.135 \pi$
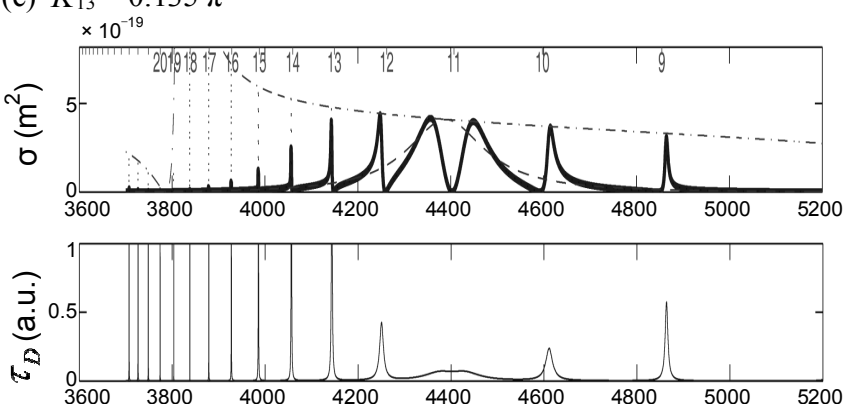

(e) $\tilde{K}_{13}=0.3 \pi$
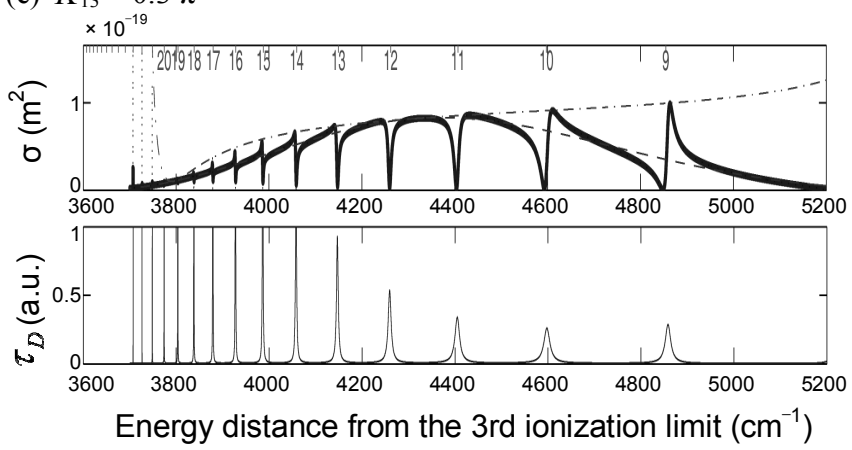

(b) $\tilde{K}_{13}=0.12 \pi$
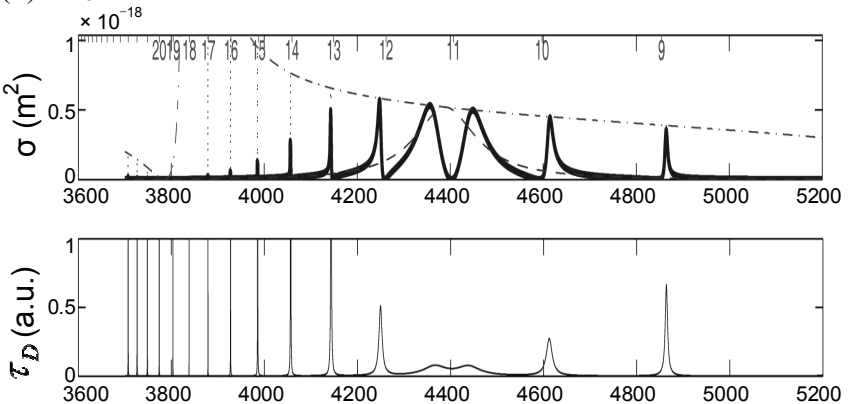

(d) $\tilde{K}_{13}=0.15 \pi$
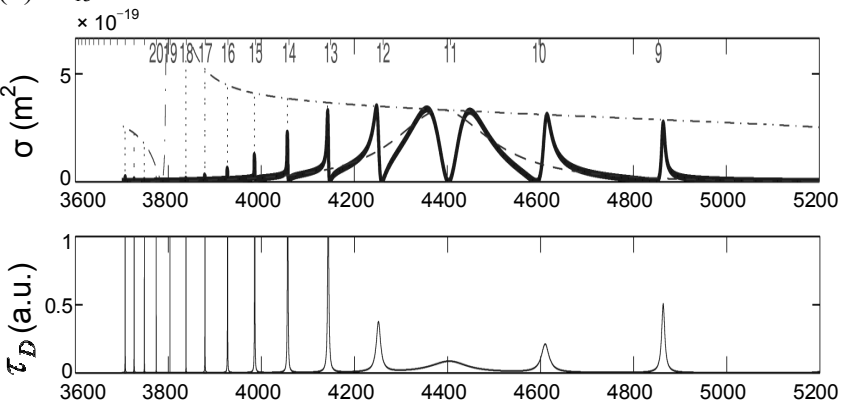

Figure 9. Transition of an interloper line into the structured background. The same parameters as the ones in Fig. 3 were used except for $\tilde{K}_{13}$, which varies from the top to the bottom graphs as $0.06 \pi, 0.12 \pi, 0.135 \pi, 0.15 \pi$ and $0.30 \pi$, respectively.

Fig. 4 shows the effect of a progressive fourfold increase in $\tilde{K}_{12}$ from $0.02 \pi$ to $5.12 \pi$. The figure confirms the resonance narrowing, the move of the resonance centers to the intermediate energies and change of $q$ to $q^{-1}$ in the case of a very strong interaction for a single Rydberg series, the phenomenon reported by Mies ${ }^{5}$ for an auto-ionized Rydberg series, and the data reported by Child ${ }^{21}$ for predissociated levels. In contrast to the complicated explanation in Mies's work, the phenomenon is so easily explained by MQDT as reported in Ref. [10]. Lane, Connerade and colleagues noticed that the $q$ reversal becomes less conspicuous as the coupling strength increases. ${ }^{4}$ This suggests that $q$ reversals could be used to access the coupling strength from the experimental data. However, the spectra show intensity enhancement in a manner that differs from spectrum to spectrum. This indicates that the spectral behavior is more complicated than they thought. We will show that all these complicated behaviors can be explained easily using our analysis tools.

The approach taken in Figs. 3 and 4 is the exploration of the dynamics through the progressive variation of one of the dynamic parameters, such as $\tilde{K}_{12}$ or $\tilde{K}_{13}$. One drawback of this app- roach is that the change in induced dynamics may not be related directly to the change in the inducing parameter and can be misunderstood. Ueda's formula and its zero and pole structures provide an analysis tool that is different in this respect. They provide the disentanglement of various dynamics involved in the formula and can identify the dynamic parameters of simple physical origin.

Before analysis, let us first describe the guiding principle of the analysis based on Ueda's formula and its zero and pole structures. The overall behavior of the auto-ionization cross section was determined from the spectrum of an interloper. The dense Rydberg series chops the interloper spectrum. Therefore, the interloper spectrum forms an envelope and serves as a structured background. The $q$ reversals of the profile indices of the lines of dense Rydberg series are determined by the roots of the quadratic equations of $\tilde{q}_{2 e f f}=0$ and poles of $\tilde{\varepsilon}_{2 e f f}$ at $-\tilde{q}_{W}$ and $-\tilde{q}_{3}$. The huge spectral enhancement from the interloper spectra, given by $\tilde{q}_{2 e f f}^{2}+1$, occurs when the peak of the dense Rydberg series lies close to the poles $\tilde{\varepsilon}_{3}=-\tilde{q}_{W}$ and $-\tilde{q}_{3}$ of $\tilde{q}_{2 \text { eff }}$ and is characterized by the sharp peak. Near $\tilde{q}_{2 e f f}=0$, no spectral 

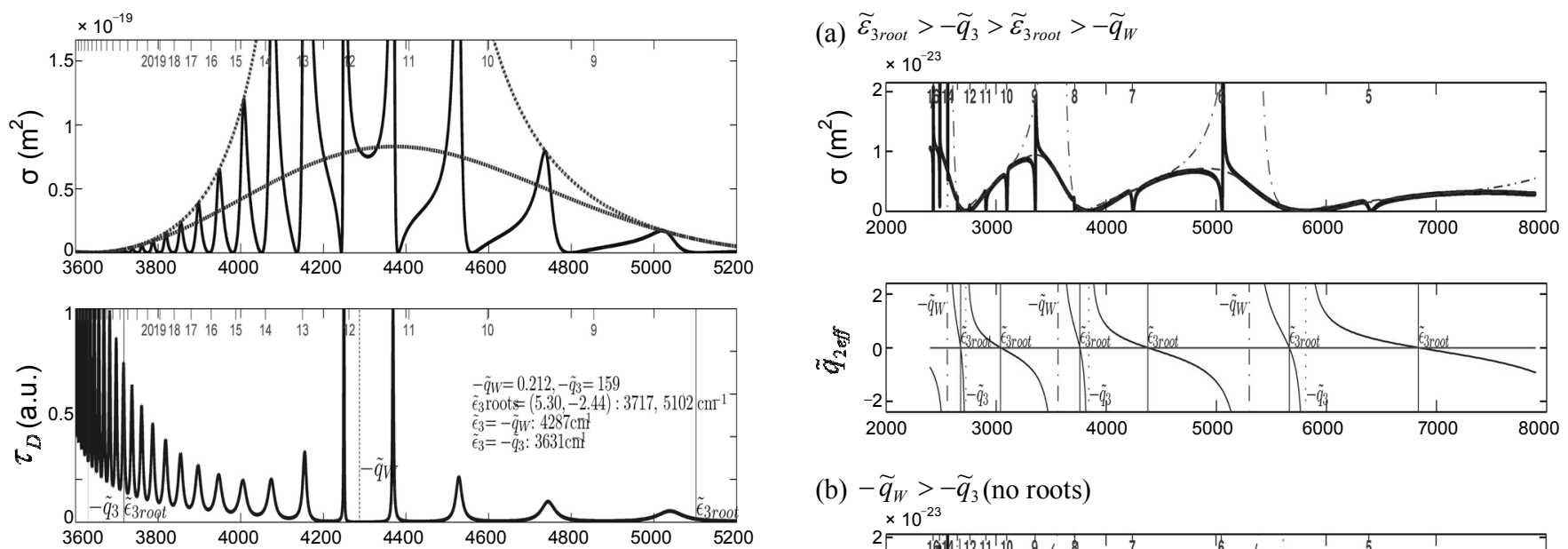

(b) $-\widetilde{q}_{W}>-\widetilde{q}_{3}$ (no roots)
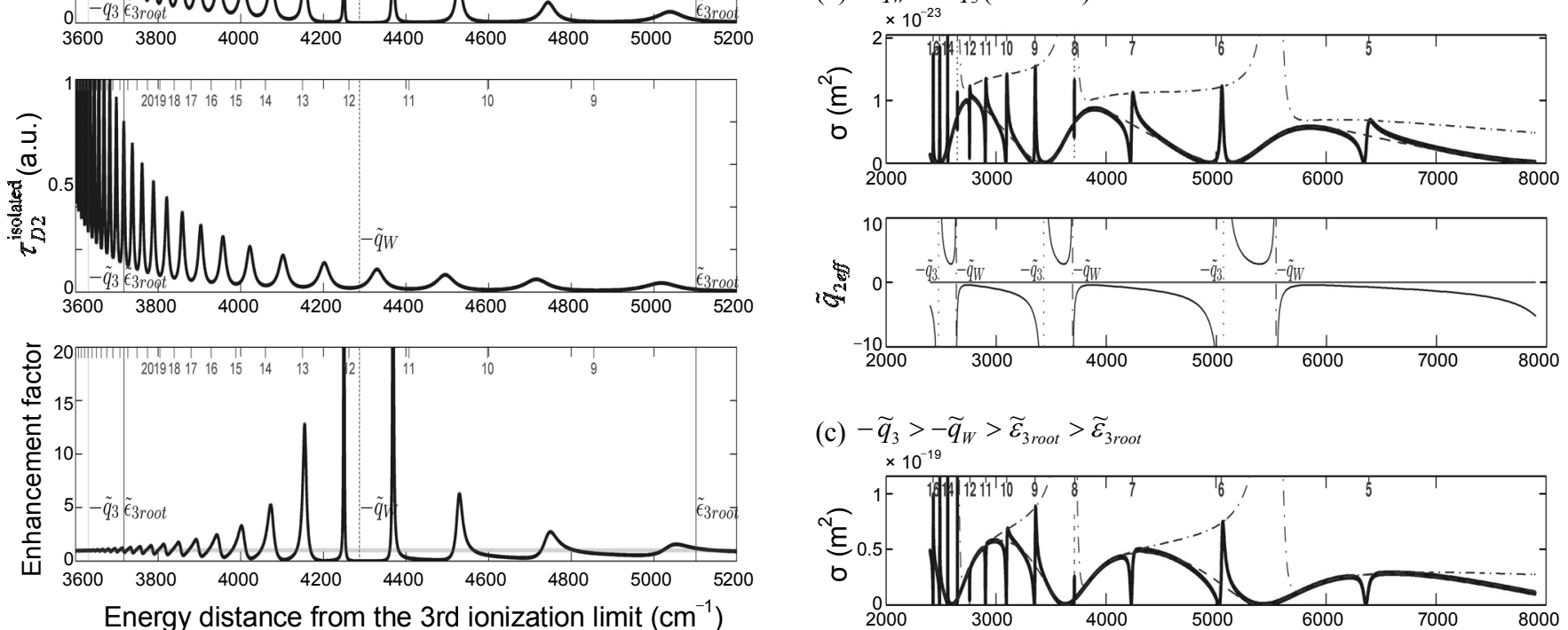

(c) $-\widetilde{q}_{3}>-\widetilde{q}_{W}>\widetilde{\varepsilon}_{3 \text { root }}>\widetilde{\varepsilon}_{3 \text { root }}$

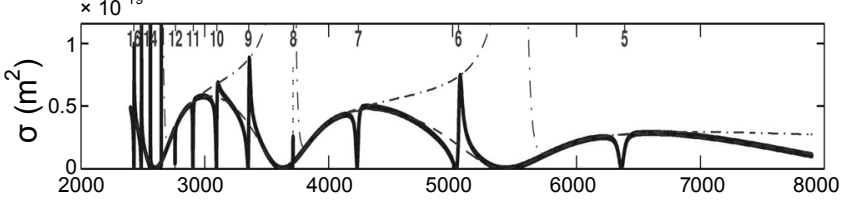

Figure 10. Analysis of the dynamics involved in time delays. $I_{i}=0$, $6500,10000 \mathrm{~cm}^{-1}(i=1,2,3) . \tilde{D}_{i}=-1,18,150$ a.u. $(i=1,2,3) . \tilde{K}_{13}=$ $0.3 \pi, \tilde{K}_{23}=0.1 \pi . \tilde{K}_{12}=0.5 \pi \cdot \mu_{i}=0(i=1,2,3)$. See Eqs. (21) and (22) for the definitions of $\tau_{D}, \tau_{D 2}^{\text {isolacd }}$ and the enhancement factor.

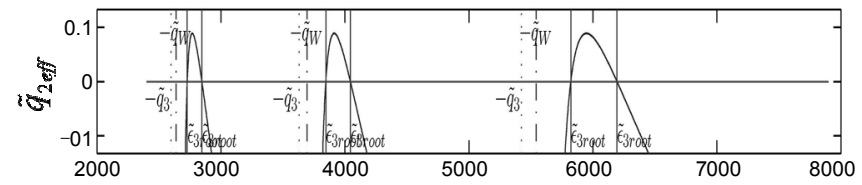

enhancement takes place and is characterized by the smooth change in the spectra.

Fig. 5 shows both the envelope graph and its enhanced version by $\tilde{q}_{2 e f f}^{2}+1$. The huge envelope consisted of several peaks at approximately $4040 \mathrm{~cm}^{-1}$ to the left-hand side of the dotted interloper resonance peak in the first spectrum that was previously interpreted as a shifted resonance peak of the interloper (see Ref. [15]). The analysis shows that this huge envelope is not the shifted one but a huge enhancement of the lines of the dense Rydberg series by the pole at $\tilde{\varepsilon}_{3}=-\tilde{q}_{W}$. The tremendous width of the envelope is just that of the structure generated by the two roots of $\tilde{q}_{2 e f f}$, at which the enhancement ends is not the width of the true interloper peak. The second spectrum of Fig. 5 is similar the first one and will be bypassed.

The third and fourth spectra have different types of $\tilde{q}_{\text {2eff }}$ graphs from the ones of the first 2 . The pole $\tilde{\varepsilon}_{3}=-\tilde{q}_{W}$ lies at the far left wing of the envelope, while most part of the envelope is governed by small $\tilde{q}_{2 e f f}$ and enhancement is suppressed. The spectra in Fig. 6 can be interpreted in the same way as for Fig. 5. One feature absent in Fig. 5 but present in Fig. 6 is the large enhancement at the resonance peak of the interloper, which is

(d) $-\widetilde{q}_{W}>\widetilde{\varepsilon}_{3 \text { root }}>\widetilde{\varepsilon}_{3 \text { root }}>-\widetilde{q}_{3}$
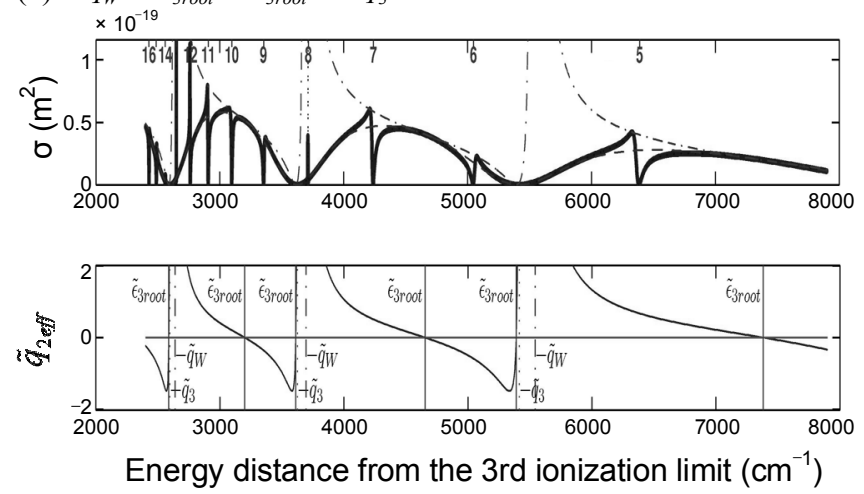

Figure 11. Four patterns corresponding to $\tilde{\varepsilon}_{3 r 1}>-\tilde{q}_{3}>\tilde{\varepsilon}_{3 r 2}>-\tilde{q}_{W}$, $-\tilde{q}_{W}>-\tilde{q}_{3}$ and imaginary roots, $-\tilde{q}_{3}>-\tilde{q}_{W}>\tilde{\varepsilon}_{3 r 1}>\tilde{\varepsilon}_{3 r 2}$ and $-\tilde{q}_{W}>$ $\tilde{\varepsilon}_{3 r 1}>\tilde{\varepsilon}_{3 r 2}>-\tilde{q}_{3}$ cases are shown. The second ionization threshold was changed to $8000 \mathrm{~cm}^{-1}$ from the previous graphs. (a) $\tilde{K}_{13}=0.4 \pi, \tilde{K}_{23}=$ $0.1 \pi$. $\tilde{K}_{12}=0.02 \pi$. (b) $\tilde{D}_{i}=1,-0.18$ and 1.5 a.u. for $i=1,2,3 . \tilde{K}_{13}=0.4 \pi$, $\tilde{K}_{23}=-0.1 \pi$. $\tilde{K}_{12}=0.02 \pi$. (c) $\tilde{D}_{i}=1,-0.18$ and -1.5 a.u. for $i=1,2,3$. $\tilde{K}_{13}=0.4 \pi, \tilde{K}_{23}=0.1 \pi$. $\tilde{K}_{12}=0.02 \pi$. (d) $\tilde{D}_{i}=1,1.8$ and -150 a.u. for $i=1,2$ and 3, respectively. $\tilde{K}_{13}=0.4 \pi, \tilde{K}_{23}=0.1 \pi$. $\tilde{K}_{12}=0.02 \pi$. 
generated by the simple pole close to the peak position of the interloper.

Fig. 5 and Fig. 6 show that the behavioral changes in the autoionization cross sections are related directly to the patterns of the $\tilde{q}_{2 \text { eff }}$ graphs. Therefore, the $\tilde{q}_{\text {2eff }}$ graphs provide a very powerful and easy-to-use tool for analyzing autoionization cross section spectra. In Fig. 5, the patterns of the $\tilde{q}_{2 \text { eff }}$ change from $\tilde{\varepsilon}_{3 \text { root }}<-\tilde{q}_{W}<\tilde{\varepsilon}_{3 \text { root }}<-\tilde{q}_{3}$ to $\tilde{\varepsilon}_{3 \text { root }}<\tilde{\varepsilon}_{3 \text { root }}<-\tilde{q}_{W}<-\tilde{q}_{3}$ between $0.10 \pi$ and $0.20 \pi$ when the value of $\tilde{K}_{13}$ increases progressively. In Fig. 6, the patterns of $\tilde{q}_{2 e f f}$ change in a reverse order from that shown in Fig. 5; from $\tilde{\varepsilon}_{3 \text { root }}<\tilde{\varepsilon}_{3 \text { root }}<-\tilde{q}_{W}<-\tilde{q}_{3}$ to $\tilde{\varepsilon}_{3 \text { root }}<-\tilde{q}_{W}<\tilde{\varepsilon}_{3 \text { root }}<-\tilde{q}_{3}$ between $0.02 \pi$ and $0.08 \pi$ when the value of $\tilde{K}_{12}$ increases progressively. Why does such a change in pattern occur? In order to understand this, let us utilize the $q$ zero surfaces in Fig. 1. Each graph in Fig. 5 and Fig. 6 is represented as a line in Fig. 1, and the increase in coupling strength $\tilde{K}_{13}$ corresponds to the movement along the $\tilde{q}_{3}$ axis in the decreasing direction. The increase in $\tilde{K}_{13}$ also brings about a decrease in the magnitude of $\tilde{q}_{W}$ as well as in $\tilde{q}_{3}$. This suggests that different $q$ zero surfaces should be used for different $\tilde{K}_{13}$. For visibility, the line will be replaced by a cylinder. Fig. 7 shows the movement of the cylinder toward the $\tilde{q}_{2}$ axis $\left(\tilde{q}_{3}=0\right)$ as $\tilde{K}_{13}$ increases in magnitude. Let $-\tilde{q}_{W}$ lie between the two roots at first as in the case of the first spectra of Fig. 5. As the cylinder moves toward the $\tilde{q}_{2}$ axis, the magnitude of $-\tilde{q}_{W}$ decreases hyperbolically whereas the upper root plane decreases linearly and the lower root plane remains constant. This means that from some point, $-\tilde{q}_{W}$ no longer lies in between the two roots. One interesting question would be the possibility of another pattern change as the point moves further, beyond the double root point and toward the $\tilde{q}_{2}$ axis. The pattern change in Fig. 6 can be explained in a similar manner.

Fig. 3 shows another interesting aspect of the overlapping resonance: the transition of an interloper spectrum from a line to a structured background. This phenomenon is discerned more conspicuously in the time delay spectra in Fig. 9. It shows that two peaks around the interloper resonance peak observed at the width of an interloper smaller than the line separation of a dense series disappear when the width becomes equal to the line separation $\left(190 \mathrm{~cm}^{-1}\right.$, corresponding to $\tilde{K}_{13}=0.13 \pi$ and $\left.v_{3}=5\right)$, and then reappears as a single peak when the width becomes larger than the line separation. The existence of a transition of the spectra of an interloper from the lines into the structured background is confirmed.

The time delay spectra revealed another interesting phenomenon, as indicated in Eq. (21). Eq. (21) shows that the time delay spectra are not decomposed into an envelope from an interloper and lines of the perturbed dense Rydberg series. It is given by the unperturbed time delays of the dense Rydberg series and the enhancement factor, which contains the effect of the interloper only indirectly. This means that the overall behavior of the time delay spectra is determined by the dense Rydberg spectra, which is in contrast to the autoionization spectra whose overall behavior is determined by the interloper spectrum. The third spectrum $\tau_{D 2}^{\text {isolated }}$ in Fig. 10 shows the typical $v^{3}$ dependence of the time delay spectra of a Rydberg series, which is due to an increase in the orbiting period around the Rydberg orbitals. It should be noted that time delay does not go to zero between the consequetive lines of the Rydberg series in the spectrum of $\tau_{D 2}^{\text {isolated }}$. This is because the value of $\tilde{K}_{12}$ is chosen as $\tilde{W}_{2}=\pi / 2$ in which the width of each peak of the dense Rydberg series is the same as the line interval. In the stronger coupling strength, intensity narrowing was also observed as in the autoionization cross section spectra in Fig. 4. Note also that $\tau_{D}$ is enhanced greatly by the enhancement factor conspicuous around the resonance peak of an interloper.

The above indicates that completely different dynamics are involved for the time delay spectra and autoionization spectra of the perturbed Rydberg spectra by an interloper whereas the dynamics of the two spectra are similar for the two channel system composed of 1 open and 1 closed channel. As stated previously, the difference in dynamics may be understood if we recall that the absorption process is instant and the dominant process is determined by the magnitude of the transition dipole moments, which is the transition to an interloper line. Since the residence time or orbiting period is proportional to $v^{3}$, it is short for the lower line of the Rydberg series, which is the case for an interloper. Therefore, the excited electron rapidly leaks into the dense series, which has a much longer orbiting period.

The $q$ reversal patterns encountered in the previous figures were two, corresponding to $\tilde{\varepsilon}_{3 \text { root }}<-\tilde{q}_{W}<\tilde{\varepsilon}_{3 \text { root }}<-\tilde{q}_{3}$ and $\tilde{\varepsilon}_{3 \text { root }}<\tilde{\varepsilon}_{3 \text { root }}<-\tilde{q}_{W}<-\tilde{q}_{3}$. All other patterns in the already mentioned 21 possible patterns could be generated. Fig. 11 shows two other patterns besides these 2 for the energy intervals containing several interloper cycles.

\section{Summary and Discussion}

A Rydberg series perturbed by an interloper is the simplest system showing complex resonance phenomena due to the overlapping resonances, and has attracted considerable research interest for more than three decades. The phase-shifted version of the multichannel quantum-defect theory (MQDT) was one of the most powerful tools to study this system. Although several groups developed the theories, diverse aspects of overlapping resonances like $q$ reversal and intensity borrowing are not well understood. Ueda's unutilized formulation that disentangles the interloper spectra from the perturbed dense Rydberg series could be quite useful for exploring such aspects of overlapping resonances. His formulation in its nascent form, though still powerful, can be made more powerful if reformulated. The pole structure in his formula, which explored following this line of thought, was found to take a simple form and allowed us to find simple quadratic equations for the zero surfaces of the line profile index $q$. The simple relations thus found allowed us to perform a deep analysis of the interesting $q$ reversal phenomena.

Using the analysis tool based on Ueda's formula and its pole structures, we performed an investigation of the interesting systems studied by Lane, Connerade and colleagues, which contained a range of overlapping resonances. This analysis tool not only provides easy access to what is occurring in the complex resonance spectra but also allows an examination of the uncharted territory of spectra with the help of the $q$ zero surfaces as a compass.

Two interesting phenomena were found from a study of the time delay spectra. One interesting phenomenon is the transition 
of the coarse intruder Rydberg series from the spectral line into the structured continuum by being dispersed onto the entire dense series. As another interesting phenomenon, overall behavior of the time delays was governed by the dense Rydberg series, which is quite different from the overall behavior of the autoionization cross sections that are governed by an interloper. This suggests that different dynamics prevail for them, which is in contrast to the two channel system, where both quantities behave in a similar manner. The dynamics obtained in the presence of overlapping resonances is as follows. The absorption process is instant and dominated by a transition to the interloper line. The process is followed by rapid leakage into the dense Rydberg series, which has a longer residence time prior to ionization than that of the interloper state. This is because the orbiting period is proportional to $v^{3}$ so that an excited electron has a shorter lifetime in the interloper state belonging to lower member of the Rydberg series. This difference in dynamics can explain the previously observed ${ }^{22}$ symmetric shapes of the time delay spectra when the autoionization spectra are highly asymmetric.

Acknowledgments. This study was supported by the Ajou university research fellowship of 2009 under contract No. 20095142COR0101S000100.

\section{References}

1. Jungen, C.; Pratt, S. T. J. Chem. Phys. 2008, 129, 164310.

2. Cohen, S.; Camus, P.; Bolovinos, A J. Phys. B 2005, 38, S1.

3. Connerade, J. P.; Lane, A. M. Rep. Prog. Phys. 1988, 51, 1439.

4. Connerade, J. P.; Lane, A. M.; Baig, M. A. J. Phys. B 1985, 18, 3507.

5. Mies, F. H. Phys. Rev. 1968, 175, 164.

6. Fano, U.; Cooper, J. W. Phys. Rev. 1965, 137, A1364.

7. Lane, A. M. J. Phys. B 1984, 17, 2213.

8. Seaton, M. J. Rep. Prog. Phys. 1983, 46, 167.

9. Fano, U.; Rau, A. R. P. Atomic Collisions and Spectra; Academic: Orlando, U.S.A., 1986.

10. Giusti-Suzor, A.; Fano, U. J. Phys. B 1984, 17, 215.

11. Cooke, W. E.; Cromer, C. L. Phys. Rev. A 1985, 32, 2725.

12. Baig, M. A. Phys. Rev. A 2009, 79, 012509.

13. Lecomte, J. M. J. Phys. B 1987, 20, 3645.

14. Cohen, S. Eur. Phys. J. D 1998, 4, 31.

15. Giusti-Suzor, A.; Lefebvre-Brion, H. Phys. Rev. A 1984, 30, 3057.

16. Wintgen, D.; Friedrich, H. Phys. Rev. A 1987, 35, 1628.

17. Ueda, K. Phys. Rev. A 1987, 35, 2484.

18. Baig, M. A.; Ahmad, S.; Connerade, J. P.; Dussa, W.; Hormes, J. Phys. Rev. A 1992, 45, 7963.

19. Smith, F. T. Phys. Rev. 1960, 118, 349.

20. Knopp, K. Theory of Functions; Dover: N.Y., 1996; Vol. II.

21. Child, M. S. J. Molec. Spectrosc. 1974, 53, 280.

22. Lee, C. W.; Kong, J. H. Bull. Korean Chem. Soc. 2009, $30,1783$. 